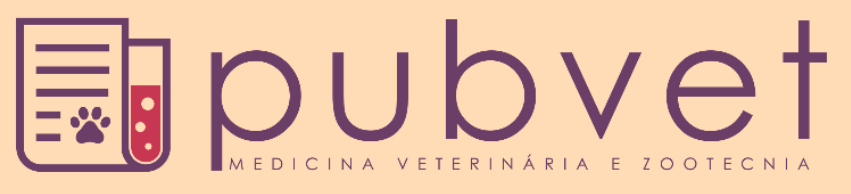

https://doi.org/10.31533/pubvet.v15n11a953.1-6

\title{
Criptorquidismo bilateral em cão: Relato de caso
}

\author{
Carla Fredrichsen Moya ${ }^{1 *}$, Marco Antônio Staudt ${ }^{2} \theta$, Fátima Maria Caetano Caldeira ${ }^{2} \theta$, \\ Gabriela Basílio Roberto ${ }^{\circ}{ }^{\circ}$, Jayme Augusto Peres ${ }^{2}{ }^{\circ}$, Adriano de Oliveira Torres Carrasco ${ }^{2}$ CD $^{\circ}$ \\ ${ }^{I}$ Docente da Universidade Estadual do Centro-Oeste - Unicentro, Departamento de Medicina Veterinária, Guarapuava - PR. \\ ${ }^{2}$ Aprimorando do Programa de Medicina Veterinária da Universidade Estadual do Centro-Oeste - Unicentro, Guarapuava - PR. \\ *Autor para correspondência, E-mail: carlafredrichsen@yahoo.com.br
}

\begin{abstract}
Resumo. O Criptorquidismo é uma anomalia congênita de testículos que possui caráter hereditário, apresentando grande importância entre as doenças que afetam a reprodução na espécie canina. O presente trabalho teve por objetivo relatar um caso de Criptorquidismo bilateral em cão, sem raça definida, com três anos de idade, atendido na Clínica Escola Veterinária Prof. Dr. Marcos Vinicius Tranquilim da Universidade Estadual do CentroOeste. Após confirmação diagnóstica da localização dos testículos intra-abdominal, por meio de ultrassonografia, realizou-se a celiotomia mediana retro-umbilical (pré-púbica) e procedeu-se a orquiectomia. $\mathrm{O}$ animal apresentou boa recuperação do procedimento cirúrgico, com a retirada dos pontos dez dias após a cirurgia, o que evidencia a eficácia do tratamento empregado nesse caso.
\end{abstract}

Palavras-chave: Canino, enfermidade reprodutiva, orquiectomia

\section{Bilateral cryptorchism in dog: Case report}

\begin{abstract}
Cryptorchidism is a congenital anomaly of the testicles that has a hereditary character, with great importance among the diseases that affect reproduction in canine species. The aim of the present study was to report a case of bilateral cryptorchidism in a three-year-old, mixed breed dog, treated at the Veterinary School Clinic Prof. Dr. Marcos Vinicius Tranquilim from the State University of the Midwest. After diagnostic confirmation of the location of the intra-abdominal testicles by means of ultrasonography, median retroumbilical (pre-pubic) celiotomy was performed and orchiectomy was accomplished. The animal had a good recovery from the surgical procedure, with the removal of the stitches ten days after the surgery, which shows the effectiveness of the treatment used in this case.
\end{abstract}

Keywords: Canine, reproductive disease, orchiectomy

\section{Introdução}

O criptorquidismo pode ser definido como uma falha na descida de um ou de ambos testículos até o escroto. Essa é a anomalia congênita mais comum de testículos, apresentando caráter hereditário e sendo de grande importância entre as enfermidades que afetam a reprodução em cães, bem como em outras espécies animais (Ettinger et al., 2002; Khan et al., 2018; Yates et al., 2003).

$\mathrm{Na}$ espécie canina, os testículos passam pelo canal inguinal cerca de três a quatro dias após o nascimento, alcançando sua posição escrotal final por volta do $14^{\circ}$ a $35^{\circ}$ dia de vida. Diante disso, o diagnóstico de criptorquidia é realizado quando um, ou ambos os testículos, não apresentaram a descida até o escroto após a oitava semana de idade (Memon \& Tibary, 2001). A incidência de Criptorquidismo é maior em cães do que em gatos, variando de $0,80 \%$ a $9,80 \%$ no cão, e com média de $1,70 \%$ no gato. Em cães, as raças mais frequentemente acometidas são Poodle, Yorkshire, Chihuahua, Boxer, Buldogue Inglês, Dachshund e Schnauzer miniatura (Ettinger et al., 2002; Morrow, 1980; Yates et al., 2003). 
Aproximadamente $75 \%$ dos casos, em cães, correspondem a pacientes com Criptorquidismo unilateral, com maior tendência de retenção do testículo direito. Embora o testículo retido seja incapaz de produzir espermatozoides, a secreção de testosterona é mantida, o que muitas vezes resulta em comportamento territorial indesejado, agressão e urina com odor forte. O testículo ectópico apresenta maior probabilidade de desenvolver processos neoplásicos, em função de alterações no mecanismo de termorregulação testicular (Kawakami et al., 1995; Mattos et al., 2000; Pena et al., 1998; Yates et al., 2003).

O diagnóstico de criptorquidismo pode ser realizado por meio de exame de tomografia computadorizada, que permite também a avaliação de infiltração metastática em linfonodos adjacentes (Stokowski et al., 2016). Todavia, devido ao custo relativamente alto e à pouca distribuição de tomógrafos na rotina veterinária, o exame ultrassonográfico é considerado uma ferramenta valiosa e mais acessível para o diagnóstico de criptorquidismo. Por meio desse exame, é possível avaliar alterações presentes no parênquima testicular e também determinar a localização dos mesmos, sendo ainda considerado um exame com boa sensibilidade para determinação do acesso cirúrgico (Felumlee et al., 2012; Tannouz et al., 2019).

O tratamento preconizado na literatura é cirúrgico, realizado por meio da orquiectomia de ambos os testículos, uma vez que essa é uma enfermidade de característica hereditária e o macho não pode ser utilizado como reprodutor (Ettinger et al., 2002; Gallagher et al., 1992; Johnston et al., 2001; Lew et al., 2005).

Os veterinários devem saber sobre a importância desta anomalia, motivando a remoção de animais com criptorquidismo unilateral da reprodução, devido à possibilidade de transmissão desta doença e em casos bilaterais ao risco do desenvolvimento de neoplasias testiculares. Diante do exposto anteriormente, o presente trabalho tem por objetivo relatar um caso de criptorquidismo bilateral em cão, sem raça definida, com três anos de idade, atendido na Clínica Escola Veterinária da Universidade Estadual do Centro-Oeste.

\section{Relato do caso}

Foi atendido na Clínica Escola Veterinária Prof. Dr. Marcos Vinicius Tranquilim da Universidade Estadual do Centro-Oeste, Guarapuava-PR, um cão com três anos de idade, sem raça definida, pesando 20 $\mathrm{kg}$, cuja queixa principal era a ausência de testículos no escroto. Na anamnese, a tutora relatou que, desde filhote, o paciente não os apresentava no escroto, porém, quando em contato com fêmeas caninas em cio, o mesmo se manifestava agitado, dificultando o manejo durante o período. Ainda, foi descrito que o animal estava vacinado e desverminado e se apresentava com normofagia, normodipsia, normoquesia e normúria.

Durante o exame físico do paciente, a frequência cardíaca era de $80 \mathrm{bpm}$, a frequência respiratória de $36 \mathrm{mpm}$, a temperatura corporal de $38,0^{\circ} \mathrm{C}$ e as mucosas estavam normocoradas. Ao se observar o escroto, verificou-se ausência de ambos os testículos (Figura 1), que também não foram detectados à palpação dos anéis inguinais. Diante dos achados e da anamnese, a suspeita clínica foi de criptorquidismo bilateral.

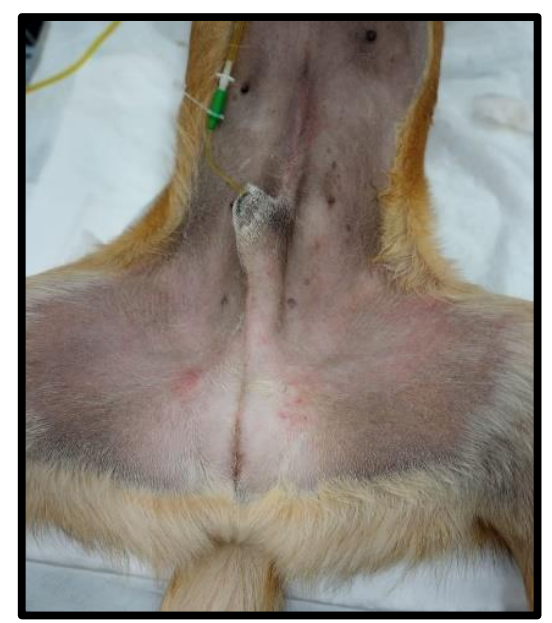

Figura 1. Macho canino, três anos de idade, sem raça definida, com ausência de ambos os testículos em escroto. 
Para diagnóstico da enfermidade, o paciente foi submetido à exame ultrassonográfico abdominal, a fim de verificar a presença e a localização de ambos os testículos. A partir do qual foi possível visibilizar a presença de duas estruturas elipsoides, com parênquima hipoecogênico em relação à gordura intraabdominal, e em ambas havia uma estrutura linear hiperecogênica central, compatível com mediastino testicular. As estruturas elipsoides correspondentes aos testículos foram visibilizadas em região imediatamente lateral direita à região cranial da vesícula urinária, e caudal e medial ao polo caudal renal direito, adjacentes à artéria aorta, chegando ao diagnóstico de Criptorquidismo bilateral. Diante disso, optouse pelo tratamento cirúrgico, contudo o mesmo só foi agendado quatro meses após a primeira consulta.

Nesse segundo momento, o paciente foi novamente encaminhado para o setor de diagnóstico por imagem para acompanhamento ultrassonográfico, com intuito de assegurar que ambos os testículos permaneciam na mesma topografia visibilizada anteriormente e para otimizar o procedimento cirúrgico. Porém, durante a avaliação, foi constatado que ambas as estruturas haviam sido deslocadas para topografia cranial à próstata e imediatamente caudal à região de trígono da vesícula urinária, sendo o testículo esquerdo mensurado com 2,28 x 1,27 cm, e o testículo direito com 2,33 x 1,43 cm (Figura 2).

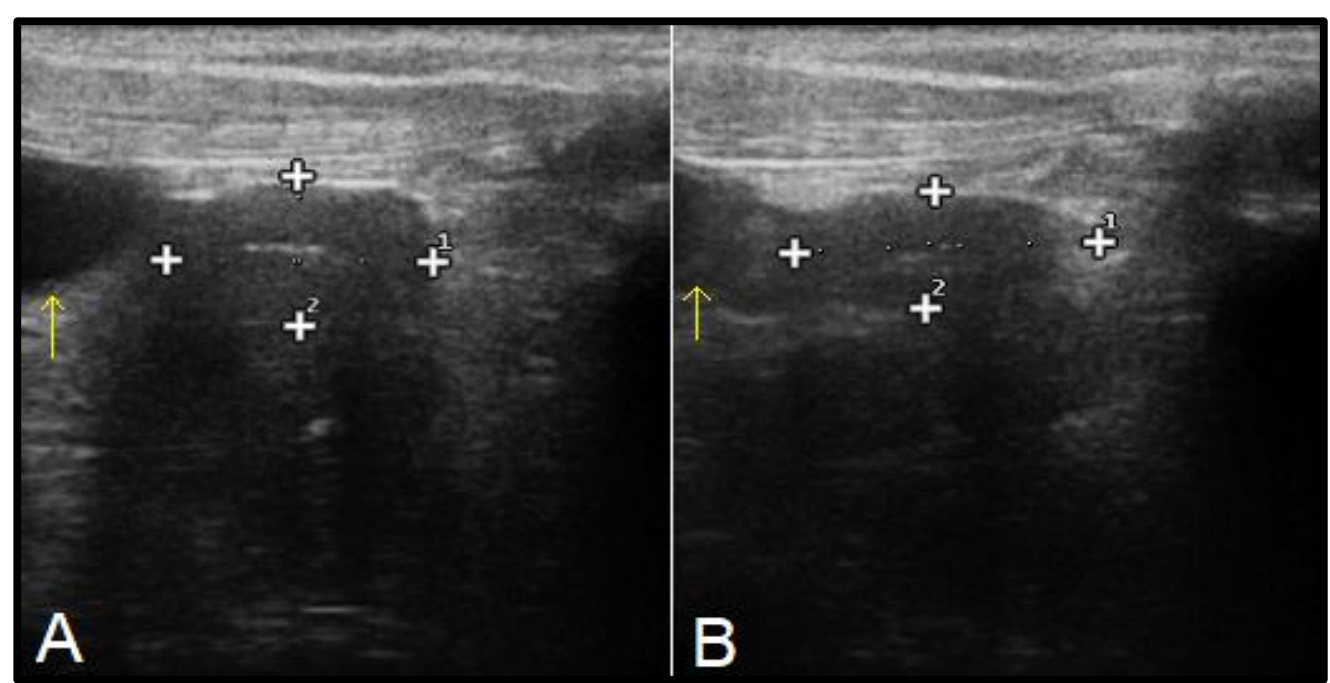

Figura 2. Imagens ultrassonográficas realizadas, por meio de transdutor linear, evidenciando a localização de ambos os testículos na cavidade abdominal. (A): É possível observar estrutura compatível com o testículo esquerdo (entre cruzes de mensuração) e porção caudal de vesícula urinária (seta amarela); (B): Estrutura compatível com testículo direito (entre cruzes de mensuração) e porção caudal de vesícula urinária (seta amarela).

Diante dos aspectos ultrassonográficos observados, dentre eles o tamanho, ecogenicidade e a topografia das estruturas, além da presença de região condizente com mediastino testicular, foi possível determinar que ambas estruturas correspondiam aos testículos, auxiliando-nos na indicação do melhor sítio cirúrgico para o procedimento.

Colheu-se o sangue para avaliação pré-operatória do animal. Nos resultados do hemograma não foram observadas alterações, e a alanina aminotransferase (ALT) e a creatinina estavam dentro do padrão de normalidade para a espécie (Weiss \& Wardrop, 2010).

$\mathrm{O}$ animal foi submetido à anestesia geral inalatória, com isoflurano, $1,5 \%$ de concentração alveolar, após medicação pré-anestésica com metadona, na dose de $0,4 \mathrm{mg} / \mathrm{kg}$, administrada por via intramuscular, e indução anestésica empregando propofol, na dose de $5 \mathrm{mg} / \mathrm{kg}$, por via endovenosa.

A técnica cirúrgica escolhida foi a de celiotomia mediana retro-umbilical (pré-púbica), iniciada por meio de incisão de pele de aproximadamente $3 \mathrm{~cm}$, divulsão do tecido subcutâneo e incisão da linha alba. Após localização do testículo esquerdo e do cordão espermático, rompeu-se o ligamento escrotal, sendo então realizadas ligadura do cordão espermático com fio absorvível sintético 3-0 e transecção distal à ligadura, com a retirada do testículo e cordão (Figura 3). Esse procedimento foi repetido no testículo direito e após conferência da hemostasia, procedeu-se a síntese da parede abdominal, sendo adotados no primeiro plano padrão Sultan, com fio absorvível sintético 2-0 e no segundo plano padrão 
contínuo com aproximação do subcutâneo, com mesmo tipo de fio. Na sequência, foi efetuada a dermorrafia, em padrão simples separado, com fio inabsorvível sintético 3-0.

No trans-operatório, foram administrados ceftriaxona, $25 \mathrm{mg} / \mathrm{kg}$ e meloxicam, $0,1 \mathrm{mg} / \mathrm{kg}$, por via endovenosa. No pós-operatório, foram prescritos tramadol, $2,4 \mathrm{mg} / \mathrm{kg}$, a cada 12 horas e, dipirona, 25 $\mathrm{mg} / \mathrm{kg}$, a cada 12 horas, ambos por cinco dias consecutivos. O curativo da ferida cirúrgica foi realizado com solução fisiológica e clorexidina a $0,2 \%$, a cada 24 horas, durante dez dias consecutivos, sendo a retirada de pontos realizada após esse período.

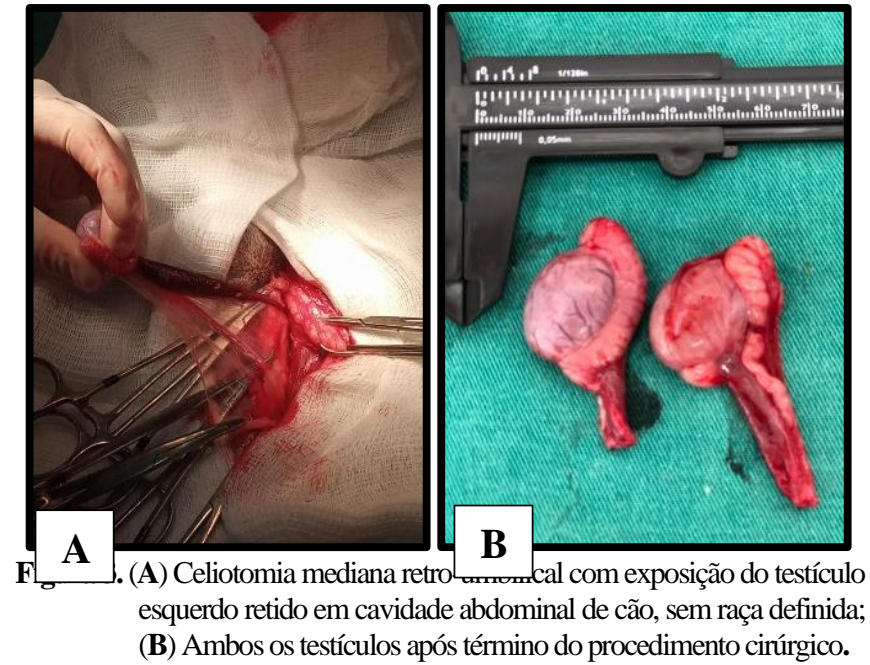

Os testículos, após o término do procedimento cirúrgico, foram palpados sendo constatada flacidez testicular, além de mensurados por meio de paquímetro, tendo o testículo esquerdo $2,5 \times 1,8 \times 1,3 \mathrm{~cm}$ e o direito 2,5 x 2,0 x 1,2 cm. Ambos foram fixados em solução formol salina tamponado e enviados ao setor de Patologia Veterinária para realização do exame histopatológico. A análise histopatológica, evidenciaram-se degeneração dos túbulos epididimários e dos túbulos seminíferos, além da ausência de atividade espermatogênica (Figura 4).

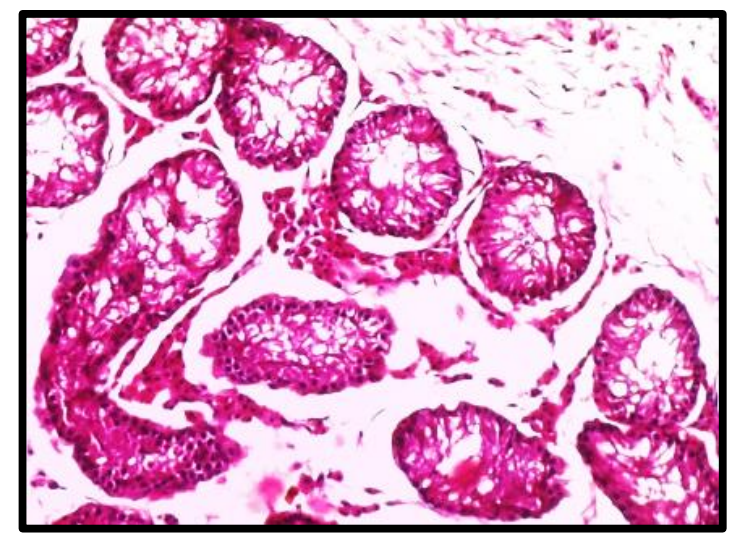

Figura 4. Corte histológico de testículo retido em cavidade abdominal de cão, sem raça definida. Presença de degeneração dos túbulos seminíferos e ausência de atividade espermatogênica. (H\&E; A:400x).

\section{Discussão}

O Criptorquidismo é mais descrito em raças de pequeno porte, como Poodle, Yorkshire, Chihuahuas, Dachshund e Schnauzers miniaturas (Ettinger et al., 2002; Morrow, 1980; Yates et al., 2003). Contudo, nesse estudo o animal era sem raça definida e pesava $20 \mathrm{~kg}$. Em quase $75 \%$ dos casos relatados, a retenção testicular é unilateral, com duas vezes mais chance de retenção do testículo direito do que o esquerdo (Kawakami et al., 1995; Mattos et al., 2000; Pena et al., 1998), e nesse caso o paciente 
apresentava ambos os testículos em cavidade abdominal, fato este menos corriqueiro na rotina médica veterinária.

Ultrassonograficamente, os testículos retidos podem estar presentes entre a margem caudal renal até a região inguinal, tendo características similares aos testículos presentes no escroto. Assim, se apresentam como estruturas elipsoides, com parênquima hipoecogênico e com estrutura linear central hiperecogênica na região do mediastino testicular (Penninck \& D'Anjou, 2011). Semelhante fato pode ser observado nesse relato, em que foi possível identificar por meio do exame ultrassonográfico duas estruturas elipsoides, com parênquima hipoecogênico e presença de mediastino.

Comumente, pacientes com Criptorquidismo podem apresentar atrofia testicular, caracterizada pela diminuição das dimensões testiculares, podendo ainda apresentar aumento ou diminuição da ecogenicidade do parênquima, sendo a diminuição da ecogenicidade mais observada (Nyland \& Mattoon, 2005). Concordando com os achados de literatura, o paciente desde estudo apresentava testículos de tamanho reduzido em comparação ao porte físico do mesmo.

Em estudo realizado por Felumlee et al. (2012) foram avaliados 34 pacientes criptorquidas encaminhados para a realização de exame ultrassonográfico, sendo possível observar que a maior casuística de localização de testículos retidos correspondia à cavidade abdominal, presentes em diferentes topografias, desde imediatamente caudal aos rins, até regiões adjacentes à vesícula urinária e ao colón. Ainda, em alguns casos, os testículos apresentavam alterações da arquitetura do parênquima, como irregularidade de contornos e alterações de ecogenicidade, porém a linha mediastinal permanecia íntegra e diferenciada. Esses dados corroboram com o observado no presente relato de caso.

Apesar de os testículos serem observados com alterações de tamanho e ecogenicidade no paciente aqui relatado, em alguns casos, é possível diagnosticar outras alterações concomitantes, dentre elas a presença de processos neoplásicos e casos de torção testicular, em que o paciente pode apresentar dor abdominal aguda e aumento de volume de epidídimo e cordão espermático (Hecht et al., 2004). Segundo Cattelan et al. (2004), o testículo ectópico é afuncional sob o ponto de vista espermatogênico, com redução no tamanho dos túbulos seminíferos e no número de células germinativas. Tal observação corrobora com os achados nesse trabalho.

O tratamento descrito na literatura é o cirúrgico, realizado por meio de orquiectomia bilateral, podendo ser realizada através de laparoscopia ou celiotomia mediana (Ettinger et al., 2002; Johnston et al., 2001; Lew et al., 2005). No presente relato, a orquiectomia bilateral foi realizada por meio de celiotomia mediana retro-umbilical (pré-púbica), após a confirmação da localização de ambos os testículos em topografia cranial à próstata e imediatamente caudal à região de trígono da vesícula urinária, realizada a partir de exame ultrassonográfico. O animal apresentou boa recuperação do procedimento cirúrgico, com a retirada dos pontos dez dias após a cirurgia, o que evidencia a escolha adequada do tratamento empregado nesse caso.

\section{Referências bibliográficas}

Cattelan, J. W., Macoris, D. G., Barnabé, P. A., Urbinati, E. C., \& Malheiros, E. B. (2004). Criptorquismo em eqüinos: aspectos clínico-cirúrgicos e determinação da testosterona sérica. Arquivo Brasileiro de Medicina Veterinária e Zootecnia, 56(2), 150-156. https://doi.org/10.1590/s0102-09352004000200003.

Ettinger, S. J., Fedlman, E. C., \& Taibo, R. A. (2002). Tratado de medicina interna veterinaria: enfermedades del perro y el gato. Manole.

Felumlee, A. E., Reichle, J. K., Hecht, S., Penninck, D., Zekas, L., Dietze Yeager, A., Goggin, J. M., \& Lowry, J. (2012). Use of ultrasound to locate retained testes in dogs and cats. Veterinary Radiology \& Ultrasound, 53(5), 581-585. https://doi.org/10.1111/j.1740-8261.2011.01943.x.

Gallagher, L. A., Freeman, L. J., Trenka-Benthin, S., \& Stoloff, D. R. (1992). Laparoscopic castration for canine cryptorchidism. Veterinary Surgery, 21, 411-412.

Hecht, S., King, R., Tidwell, A. S., \& Gorman, S. C. (2004). Ultrasound diagnosis: intra-abdominal torsion of a non-neoplastic testicle in a cryptorchid dog. Veterinary Radiology \& Ultrasound, 45(1), 58-61. https://doi.org/10.1111/j.1740-8261.2004.04009.x. 
Johnston, S. D., Olson, M. V, Johnston, P. S. S. D., Kustritz, M. V. R., \& Olson, P. N. S. (2001). Canine and feline theriogenology. WB Saunders.

Kawakami, E., Tsutsui, T., Saito, S., Kakimoto, T., \& Ogasa, A. (1995). Changes in peripheral plasma luteinizing hormone and testosterone concentrations and semen quality in normal and cryptorchid dogs during sexual maturation. Laboratory Animal Science, 45(3), 258-263.

Khan, F. A., Gartley, C. J., \& Khanam, A. (2018). Canine cryptorchidism: An update. Reproduction in Domestic Animals, 53(6), 1263-1270.

Lew, M., Jałyński, M., Kasprowicz, A., \& Brzeski, W. (2005). Laparoscopic cryptorchidectomy in dogs-report of 15 cases. Polish Journal of Veterinary Sciences, 8(3), 251-254.

Mattos, M. R. F., Simões-Mattos, L., \& Domingues, S. F. S. (2000). Cryptorchidism in dog. Ciência Animal, 10(1), 61-70.

Memon, M., \& Tibary, A. (2001). Canine and feline cryptorchidism. In P. W. Concannon, G. England, \& J. Verstegen (Eds.), Recent advances in small animal reproduction (pp. 1-4).

Morrow, D. A. (1980). Current therapy in theriogenology: diagnosis, treatment, and prevention of reproductive diseases in animals. Saunders.

Nyland, T. G., \& Mattoon, J. S. (2005). Ultra-som diagnóstico em pequenos animais. Roca.

Pena, F. J., Anel, L., Dominguez, J. C., Alegre, B., Alvarez, M., Celorrio, I., \& Anel, E. (1998). Laparoscopic surgery in a clinical case of seminoma in a cryptorchid dog. The Veterinary Record, 142(24), 671-672. https://doi.org/10.1136/vr.142.24.671.

Penninck, D. G., \& D’Anjou, M. A. (2011). Atlas de ultrassonografia de Pequenos animais (p. 513p.). Guanabara Koogan.

Stokowski, S., Ruth, J., Lanz, O., \& Ziglioli, V. (2016). Computed tomographic features in a case of bilateral neoplastic cryptorchidism with suspected torsion in a dog. Frontiers in Veterinary Science, 3, 1-5. https://doi.org/10.3389/fvets.2016.00033.

Tannouz, V. G. S., Mamprim, M. J., Lopes, M. D., Santos-Sousa, C. A., Junior, P. S., Babinski, M. A., \& Abidu-Figueiredo, M. (2019). Is the right testis more affected by cryptorchidism than the left testis? An ultrasonographic approach in dogs of different sizes and breeds. Folia Morphologica, 78(4), 847-852. https://doi.org/10.5603/FM.a2019.0022.

Weiss, D. J., \& Wardrop, J. K. (2010). Schalm's Veterinary Hematology.

Yates, D., Hayes, G., Heffernan, M., \& Beynon, R. (2003). Incidence of cryptorchidism in dogs and cats. Veterinary Record, 152(16), 502-504. https://doi.org/10.1136/vr.152.16.502.

Histórico do artigo:

Recebido: 16 de junho de 2021

Aprovado: 21 de julho de 2021
Licenciamento: Este artigo é publicado na modalidade Acesso Aberto sob a licença Creative Commons Atribuição 4.0 (CC-BY 4.0), a qual permite uso irrestrito, distribuição, reprodução em qualquer meio, desde que o autor e a fonte sejam devidamente creditados. 